\title{
Econophysics: What can physicists contribute to economics?
}

\author{
Parameswaran Gopikrishnan*, Luís A. Nunes Amaral*, \\ Yanhui Liu*, Martin Meyer*, Vasiliki Plerou ${ }^{\dagger *}$, \\ Bernd Rosenow ${ }^{\ddagger}$, and H. Eugene Stanley*. \\ * Center for Polymer Studies ${ }^{1}$ and Department of Physics, \\ Boston University, Boston MA 02215, USA. \\ $\dagger$ Department of Physics, Boston College, Chestnut Hill, MA 02617, USA. \\ $\ddagger$ Institut für Theoretische Physik, Universität zu Köln, D-50937 Köln, Germany.
}

\begin{abstract}
In recent years, a considerable number of physicists have started applying physics concepts and methods to understand economic phenomena. The term "Econophysics" is sometimes used to describe this work. Economic fluctuations can have many repercussions, and understanding fluctuations is a topic that many physicists have contributed to in recent years. Further, economic systems are examples of complex interacting systems for which a huge amount of data exist and it is possible that the experience gained by physicists in studying fluctuations in physical systems might yield new results in economics. Much recent work in econophysics is focussed on understanding the peculiar statistical properties of price fluctuations in financial time series. In this talk, we discuss three recent results. The first result concerns the probability distribution of stock price fluctuations. This distribution decreases with increasing fluctuations with a power-law tail well outside the Lévy stable regime and describes fluctuations that differ by as much as 8 orders of magnitude. Further, this non stable distribution preserves its functional form for fluctuations on time scales that differ by 3 orders of magnitude, from $1 \mathrm{~min}$ up to approximately 10 days. The second result concerns the accurate quantification of volatility correlations in financial time series. While price fluctuations themselves have rapidly decaying correlations, the volatility estimated by using either the absolute value or the square of the price fluctuations has correlations that decay as a power-law and persist for several months. The third result bears on the application of random matrix theory to understand the correlations among price fluctuations of any two different stocks. We compare the statistics of the cross-correlation matrix constructed from price fluctuations of the leading 1000 stocks and a matrix with independent random elements, i.e., a random matrix. Contrary to first expectations, we find little or no deviation from the universal predictions of random matrix theory for all but a few of the largest eigenvalues of the cross-correlation matrix.
\end{abstract}

1) The Center for Polymer Studies is supported by the National Science Foundation.

CP511, Unsolved Problems of Noise and Fluctuations, edited by D. Abbott and L. B. Kish (C) 2000 American Institute of Physics $1-56396-826-6 / 00 / \$ 17.00$ 


\section{INTRODUCTION}

The analysis of financial data using concepts and methods developed for physical systems has a long tradition [1-4] and has recently attracted the interest of physicists [5-9]. Possible reasons for this interest include the scientific challenge of understanding the dynamics of a strongly fluctuating complex system with a large number of interacting elements. Moreover economic fluctuations could have many repercussions and understanding fluctuations in physical systems is a topic where many physicists have contributed. In addition, it is possible that the experience gained by studying fluctuations in physical systems might yield new results in economics.

One can ask how physicists can contribute to the search for solutions to the puzzles posed by modern economics that economists themselves have not yet solved? One approach - in the spirit of experimental physics - is to begin empirically, with real data that one can analyze in some detail, but without prior models. In economic systems such as financial markets, one has available a great deal of real data. Moreover, if one has at one's disposal the tools of statistical physics and the computing power to carry out any number of approaches, this abundance of data is to great advantage. Thus, for many physicists, studying the economy means studying a wealth of data on a strongly fluctuating complex system. Indeed, physicists in increasing numbers are finding problems posed by economics sufficiently challenging to engage their attention $[10-26]$.

Recent studies attempt to uncover and explain the peculiar statistical properties of financial time series such as stock prices, stock market indices or currency exchange rates. The dynamics of financial markets is difficult to understand not only because of the complexity of its internal elements but also due to the many intractable external factors acting on it, which may differ from market to market. Remarkably, the statistical properties of certain observables appear to be similar for quite different markets [31], consistent with the possibility that there may exist "universal" mechanisms.

The most challenging difficulty in the study of financial markets is that the nature of the interactions between the different elements comprising the system is unknown, as is the way in which external factors affect it. Therefore, as a starting point, one may resort to empirical studies to help uncover the regularities or "empirical laws" that may govern financial markets [27]. The interactions between the different elements comprising financial markets generate many observables such as the transaction price, the share volume traded, the trading frequency, and the values of market indices. Recent empirical studies are based on the analysis of price fluctuations. This talk reviews recent results on (a) the distribution of stock price fluctuations and its scaling properties, (b) time-correlations in financial time series, and (c) correlations among the price fluctuations of different stocks. Space limitations restrict us to focusing mainly on our group's work; a more balanced 
account can be found in two recent books [5,6], other articles in these proceedings, and two other recent international conferences $[7,9]$. Recent work in this field also focuses on applications such as risk control, derivative pricing, and portfolio selection [28], which shall not be discussed in this talk. The interested reader should consult, for example, Refs. [5,6,29].

\section{DISTRIBUTION OF PRICE FLUCTUATIONS}

The recent availability of "high frequency" data allows one to study economic time series on a wide range of time scales varying from seconds up to a few months. For example, our recent work $[18,40,41]$ involves the analysis of the S\&P 500 index, an index of the New York Stock Exchange that consists of 500 companies representative of the US economy. It is a market-value (stock price times number of shares outstanding) weighted index, with each stock's weight in the index proportionate to its market value [18]. The S\&P 500 index is one of the most widely used benchmarks of U.S. equity performance. We analyzed high frequency data for the 13-year period 1984-96 with a recording frequency of one minute or shorter and the daily records for the 35-year period 1962-96 (Fig. 1a).

The S\&P 500 index $Z(t)$ from 1962-96 has an overall upward drift - interrupted by drastic events such as the market crash of October 19, 1987 (Fig. 1a). One analyzes the difference in logarithm of the index, often called the return $G(t)=$ $\log _{e} Z(t+\Delta t)-\log _{e} Z(t)$, where $\Delta t$ is the time scale investigated (Fig. 1b). One only counts the number of minutes during the opening hours of the stock market. It
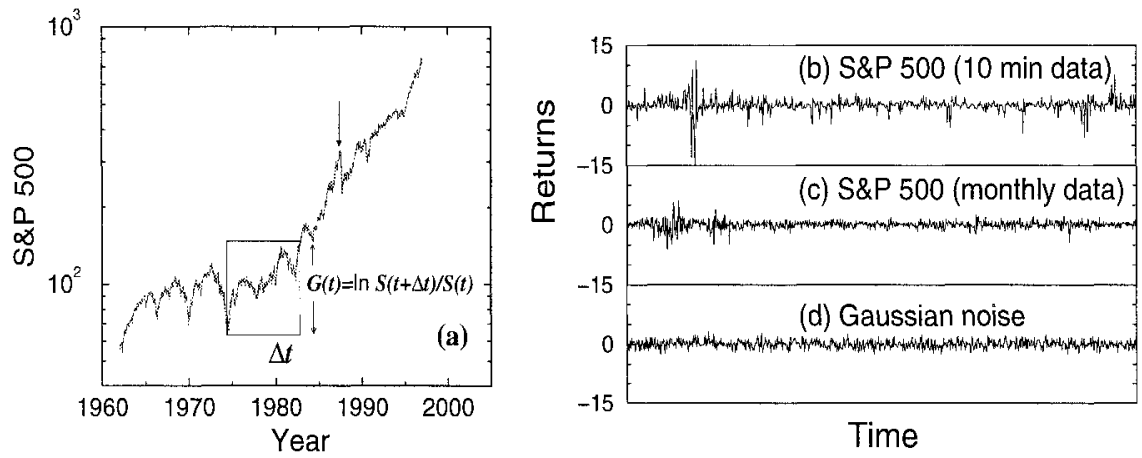

FIGURE 1. (a) The daily records of the S\&P 500 index for the 35-year period 1962-96 on a linear-log scale. Note the large jump which occured during the market crash of October 19, 1987. Sequence of $(b) 10 \mathrm{~min}$ returns and (c) 1 month returns of the S\&P 500 index, normalized to unit variance. (d) Sequence of i.i.d. Gaussian random variables with unit variance, which was proposed by Bachelier as a model for stock returns [1]. For all 3 panels, there are 850 events -i.e., in panel (b) 850 minutes and in panel (c) 850 months. Note that, in contrast to (b) and (c), there are no large events in (d). 
is apparent from Fig. $1 \mathrm{~b}$ that when one analyzes returns on short time scales, large events are much more likely to occur, in contrast to a sequence of Gaussian distributed random numbers of the same variance (Fig. 1d). As one analyzes returns on larger time scales, this difference is apparently much less pronounced (Fig. 1c). In order to understand this process, one starts by analyzing the probability distribution of returns on a given time scale $\Delta t$, which in our study, varies from $1 \mathrm{~min}$ up to a few months.

The nature of the distribution of price fluctuations in financial time series is a long standing open problem in finance which dates back to the turn of the century. In 1900, Bachelier proposed the first model for the stochastic process of returnsan uncorrelated random walk with independent, identically Gaussian distributed (i.i.d) random variables [1]. This model is natural if one considers the return over a time scale $\Delta t$ to be the result of many independent "shocks", which then lead by the central limit theorem to a Gaussian distribution of returns [1]. However, empirical studies $[4,18,19]$ show that the distribution of returns has pronounced tails in striking contrast to that of a Gaussian. Despite this empirical fact, the Gaussian assumption for the distribution of returns is widely used in theoretical finance because of the simplifications it provides in analytical calculation; indeed, it is one of the assumptions used in the classic Black-Scholes option pricing formula [30].

In his pioneering analysis of cotton prices, Mandelbrot observed that in addition to being non-Gaussian, the process of returns shows another interesting property: "time scaling" - that is, the distributions of returns for various choices of $\Delta t$, ranging from 1 day up to 1 month have similar functional forms [4]. Motivated by (i) pronounced tails, and (ii) a stable functional form for different time scales, Mandelbrot [4] proposed that the distribution of returns is consistent with a Lévy stable distribution $[2,3]$.

Conclusive results on the distribution of returns are difficult to obtain, and require a large amount of data to study the rare events that give rise to the tails. More recently, the availability of high frequency data on financial market indices, and the advent of improved computing capabilities, has facilitated the probing of the asymptotic behavior of the distribution. For example, Mantegna and Stanley [18] analyzed approximately 1 million records of the S\&P 500 index. They report that the central part of the distribution of S\&P 500 returns appears to be well fit by a Lévy distribution, but the asymptotic behavior of the distribution of returns shows faster decay than predicted by a Lévy distribution. Hence, Ref. [18] proposed a truncated Lévy distribution - a Lévy distribution in the central part followed by an approximately exponential truncation - as a model for the distribution of returns. The exponential truncation ensures the existence of a finite second moment, and hence the truncated Lévy distribution is not a stable distribution $[32,33]$. The truncated Lévy process with i.i.d. random variables has slow convergence to Gaussian behavior due to the Lévy distribution in the center, which could explain the observed time scaling for a considerable range of time scales [18].

Recent, studies [34,35] on considerably larger time series using larger databases 
show quite different asymptotic behavior for the distribution of returns. Our recent work [34] analyzed three different data bases covering securities from the three major US stock markets. In total, we analyzed approximately 40 million records of stock prices sampled at $5 \mathrm{~min}$ intervals for the 1000 leading US stocks for the 2-year period 1994-95 and 35 million daily records for 16,000 US stocks for the 35-year period 1962-96. We study the probability distribution of returns (Fig. 2(a,b,c)) for individual stocks over a time interval $\Delta t$, where $\Delta t$ varies approximately over a
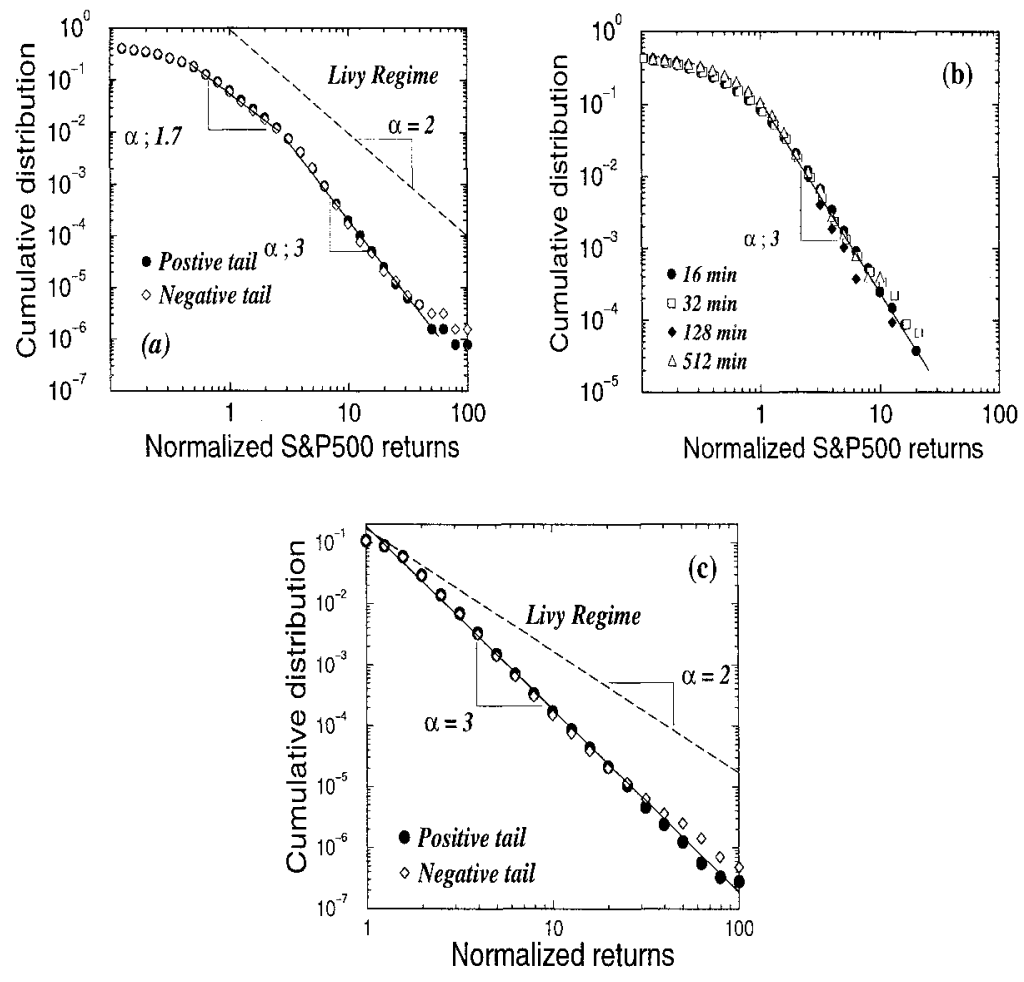

FIGURE 2. (a) Log-log plot of the cumulative distribution of the normalized 1 min returns for the S\&P 500 index. Power-law regression fits in the region $3 \leq g \leq 50$ yield $\alpha=2.95 \pm 0.07$ (positive tail), and $\alpha=2.75 \pm 0.13$ (negative tail). For the region $0.5 \leq g \leq 3$, regression fits give $\alpha=1.6 \pm 0.1$ (positive tail), and $\alpha=1.7 \pm 0.1$ (negative tail). (b) Log-log plot of the cumulative distribution of normalized returns of the S\&P 500 index. The positive tails are shown for $\Delta t=16,32,128,512$ mins. Power-law regression fits yield estimates of the asymptotic power-law exponent $\alpha=2.69 \pm 0.04, \alpha=2.53 \pm 0.06, \alpha=2.83 \pm 0.18$ and $\alpha=3.39 \pm 0.03$ for $\Delta t=16,32,128$ and 512 mins, respectively. (c) The positive and negative tails of the cumulative distribution of the normalized returns of the 1000 largest companies in the TAQ database for the 2-year period 1994-1995. The solid line is a power-law regression fit in the region $2 \leq x \leq 80$. 
factor of $10^{4}$-from $1 \mathrm{~min}$ up to more than 1 month. We also conduct a parallel study of the S\&P 500 index.

Our key finding is that the cumulative distribution of returns for both individual companies (Fig. 2c) and the S\&P 500 index (Fig. 2a) can be well described by a power law asymptotic behavior, characterized by an exponent $\alpha \approx 3$, well outside the stable Lévy regime $0<\alpha<2$. Further, it is found that the distribution, although not a stable distribution, retains its functional form for time scales up to approximately 16 days for individual stocks and approximately 4 days for the $\mathrm{S} \& \mathrm{P}$ 500 index, Fig. 2b. For larger time scales our results are consistent with breakdown of scaling behavior, i.e., convergence to Gaussian [34]. Similar results have also been found for currency exchange data [35].

\section{CORRELATIONS IN FINANCIAL TIME SERIES}

In addition to the probability distribution, an aspect of equal importance for the characterization of any stochastic process is the quantification of correlations. Studies of the autocorrelation function of the returns show exponential decay with characteristic decay times of only 4 min [36] consistent with the efficient market hypothesis [37]. This is paradoxical, for in the previous section, we have seen that the distribution of returns, in spite of being a non-stable distribution, preserves its shape for a wide range of $\Delta t$. Hence, there has to be some sort of correlations or dependencies that prevent the central limit theorem to take over sooner and preserve the scaling behavior.

Indeed, lack of linear correlation does not imply independent returns, since there may exist higher-order correlations. Recently, Liu and his collaborators found that the amplitude of the returns, the absolute value or the square - closely related to what is referred to in economics as the volatility [38] - shows long-range correlations [21,23,39-42] with persistence [43] up to several months, Fig. 3(a,b). They analyzed the correlations in the absolute value of the returns $[40,41]$ of the S\&P 500 index using traditional correlation function estimates, power spectrum and the recently-developed detrended fluctuation analysis (DFA). All the three methods show the existence of power-law correlations with a cross-over at approximately 1.5 days. For the S\&P 500 index, DFA estimates for the exponents characterizing the power law correlations are $\alpha_{1}=0.66$ for short time scales smaller than $\approx$ 1.5 days and $\alpha_{2}=0.93$ for longer time scales up to a year, Fig. 3b. For individual companies, the same methods yield $\alpha_{1}=0.60$ and $\alpha_{2}=0.74$, respectively. The power spectrum gives consistent estimates of the two power-law exponents, Fig. 3a.

The long memory in the amplitude of returns suggests that it is useful to define a subsidiary process, referred to as the volatility. Volatility of a certain stock measures how much it is likely to fluctuate. It can also be related to the amount of information arriving at any time. The volatility can be estimated for example by the local average of the absolute values or the squares of the returns. In their recent work on the statistical properties of volatility Liu et. al. $[41,42]$ show that the 

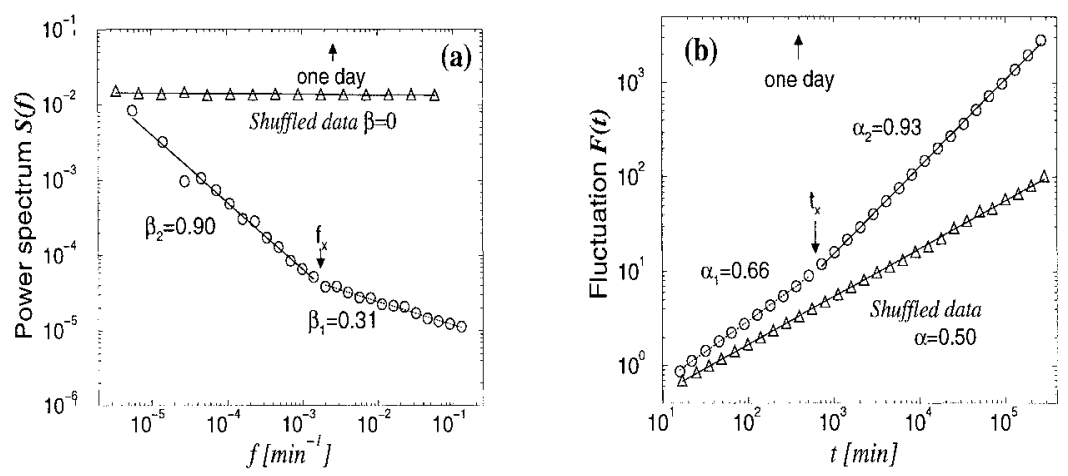

FIGURE 3. Plot of (a) the power spectrum $S(f)$ and (b) the detrended fluctuation analysis $F(t)$ of the absolute values of returns $g(t)$, after detrending the daily pattern $[40,41]$ with the sampling time interval $\Delta t=1 \mathrm{~min}$. The lines show the best power law fits ( $R$ values are better than 0.99) above and below the crossover frequency of $f_{\times}=(1 / 570) \mathrm{min}^{-1}$ in (a) and of the crossover time, $t_{\times}=600 \mathrm{~min}$ in (b). The triangles show the power spectrum and DFA results for the "control", i.e., shuffled data.

volatility correlations show asymptotic $1 / f$ behavior [40-42]. Using the same data bases as above, Liu and his collaborators also study the cumulative distribution of volatility $[40,42]$ and find that it is consistent with a power-law asymptotic behavior, characterized by an exponent $\mu \approx 3$, just the same as that for the distribution of returns. For individual companies also, one finds a similar power law asymptotic behavior [41]. In addition, it is also found that the volatility distribution scales for a range of time intervals just as the distribution of returns.

\section{CORRELATIONS AMONG DIFFERENT UNITS}

Recently, the problem of understanding the correlations among the returns of different stocks has been addressed by applying methods of random matrix theory to the cross-correlation matrix $[46,47]$. Aside from scientific interest, the study of correlations between the returns of different stocks is also of practical relevance in quantifying the risk of a given portfolio [28]. Consider, for example, the equaltime correlation of stock returns for a given pair of companies. Since the market conditions may not be stationary, and the historical records are finite, it is not clear if a measured correlation of returns of two stocks is just due to "noise" or genuinely arises from the interactions among the two companies. Moreover, unlike most physical systems, there is no "algorithm" to calculate the "interaction strength" between two companies (as there is for, say, two spins in a magnet). The problem is that although every pair of companies should interact either directly or indirectly, 
the precise nature of interaction is unknown.

In some ways, the problem of interpreting the correlations between individual stock-returns is reminiscent of the difficulties experienced by physicists in the fifties, in interpreting the spectra of complex nuclei. Large amounts of spectroscopic data on the energy levels were becoming available but were too complex to be explained by model calculations because the exact nature of the interactions were unknown. Random matrix theory (RMT) was developed in this context, to deal with the statistics of energy levels of complex quantum systems [48-50]. With the minimal assumption of a random Hamiltonian, given by a real symmetric matrix with independent random elements, a series of remarkable predictions were made and successfully tested on the spectra of complex nuclei [48]. RMT predictions represent an average over all possible interactions [49]. Deviations from the universal predictions of RMT identify system-specific, non-random properties of the system under consideration, providing clues about the underlying interactions [50]

Recently, Plerou and her collaborators analyzed the cross-correlation matrix $\mathrm{C}$ $\equiv C_{i j} \equiv\left\langle G_{i} G_{j}\right\rangle-\left\langle G_{i}\right\rangle\left\langle G_{j}\right\rangle / \sigma_{i} \sigma_{j}$ of the returns at 30-minute intervals of the largest 1000 US stocks for the 2-year period 1994-95. They analyze the statistical properties of $C$ by applying techniques of random matrix theory (RMT) [46,47]. First, they test the eigenvalue statistics of the cross-correlation matrix for universal properties of real symmetric random matrices such as the Wigner surmise for the eigenvalue spacing distribution and eigenvalue correlations. Remarkably, they find that eigenvalue statistics of the correlation matrix agree well with the universal predictions of random matrix theory for real symmetric random matrices, in contrast to our naive expectations for a strongly interacting system.

Deviations from RMT predictions represent genuine correlations. In order to investigate deviations, we compute the distribution of the eigenvalues of the $C$ and compare with the prediction [46] for uncorrelated time series [51]. We find that the statistics of all but a few of the largest eigenvalues in the spectrum of $C$ agree with the predictions of random matrix theory, but there are deviations for a few of the largest eigenvalues $[46,47]$. The deviations of the largest few eigenvalues from the random matrix result are also found when one analyzes the distribution of eigenvector components. Specifically, the largest eigenvalue which deviates significantly (25 times larger than random matrix bound) has almost all components participating equally and thus represents the correlations that pervade through the entire market. This result is in agreement with the results of Laloux and collaborators [46] for the eigenvalue distribution of $\mathrm{C}$ on a daily time scale.

\section{CONCLUSIONS AND OPEN QUESTIONS}

Econophysics is a field wherein questions are as difficult to pose as answers. The empirical results shown above clearly beckon explanation. For example, in first two sections, we have looked mainly at two empirical results: (i) the distribution of fluctuations, which shows a power law behavior well outside the stable Lévy 
regime, and yet preserves its shape-scales - for a range of time scales and (ii) the long range correlations in the amplitude of price fluctuations. How are the two related?

Previous explanations of scaling relied on Lévy stable [4] and exponentiallytruncated Lévy processes $[6,18]$. However, the empirical data that we analyze are not consistent with either of these two processes. In order to confirm that the scaling is not due to a stable distribution, one can randomize the time series of 1 min returns, thereby creating a new time series which contains statisticallyindependent returns. By adding up $n$ consecutive returns of the shuffled series, one can construct the $n$ min returns. Both the distribution and its moments show a rapid convergence to Gaussian behavior with increasing $n$, showing that the time dependencies, specifically volatility correlations are intimately connected to the observed scaling behavior [34].

Using the statistical properties summarized above, can we attempt to deduce a statistical description of the process which gives rise to this output? For example, the standard ARCH model $[27,44]$ reproduces the power-law distribution of returns; however it assumes finite memory on past events and hence is not consistent with long-range correlations in volatility. On the other hand, the distribution of volatility and that of returns which have similar asymptotic behavior, however support the central ARCH hypothesis that $g(t)=\epsilon v(t)$, where $\epsilon$ is an i.i.d. Gaussian random variable independent of the volatility $v(t)$, and $g(t)$ denotes the returns. A consistent statistical description may involve extending the traditional ARCH model to include long-range volatility correlations [45].

A more fundamental question would be to understand the above results starting from a microscopic setting. Researchers have also studied microscopic models that might give rise to the empirically observed statistical properties of returns $[5,10]$. For example, Lux and Marchesi [10] recently simulated a microscopic model of financial markets with two types of traders, what they refer to as 'fundamentalist' and 'noise' traders. Their results reproduce the power-law tail for the distribution of returns and also the long range correlations in volatility.

In the last section, we found evidence for different modes of correlations between different companies. For example, the largest eigenvalue of the cross-correlation matrix showed correlations that pervade the entire market. Could it be that the above observed scaling properties are related to how correlations propogate from one unit to the other such as occur in critical phenomena? Researchers have studied economic data from the physics perspective of a complex system with each unit depending on the other. Specifically, the possibility that all the companies in a given economy might interact, more or less, like a spin glass. In a spin glass, each spin interacts with every other spin-but not with the same coupling and not even with the same sign. For example, if the stock price of a given business firm A decrease by, e.g., $10 \%$, this will have an impact in the economy. Some of these will be favorable - firm B, which competes with A, may experience an increase in market share. Others will be negative - service industries that provide personal services for firm A employees may experience a drop-off in sales as employee salaries will surely 
decline. There must be positive and negative correlations for almost any economic change. Can we view the economy as a complicated spin glass?

To approch this problem, M. H. R. Stanley and M. A. Salinger first located and secured a database - called COMPUSTAT - that lists the annual sales of every firm in the United States. With this information, M. H. R. Stanley and co-workers calculated histograms of how firm sizes change from one year to the next [52]. They find that the distribution of growth rates of firm sales has the same functional form regardless of industry or market capitalization. Moreover, the width of these distributions decrease with increasing sales as a power-law with an exponent approximately 1/6. Recently, similar statistical properties were found for the GDP of countries [53] and for university research fundings [54]. Hence, it is not impossible to imagine that there are some very general principles of complex organizations at work here, because similar empirical laws appear to hold for data on a range of systems that at first sight might not seem to be so closely related. Buldyrev models this firm structure as an approximate Cayley tree, in which each subunit of a firm reacts to its directives from above with a certain probability distribution [55]. More recently, Amaral et al. [56] have proposed a microscopic model that reproduces both the exponent and the distribution function. Takayasu and Okuyama [57] extended the empirical results to a wide range of countries.

We conclude by thanking all our collaborators and colleagues from whom we learned a great deal. These include the researchers and faculty visitors to our research group with whom we have enjoyed the pleasure of scientific collaboration. Those whose research provided the basis of this short report include: S. V. Buldyrev, D. Canning, P. Cizeau, X. Gabaix, S. Havlin, P. Ch. Ivanov, R. N. Mantegna, C.-K. Peng, M. A. Salinger, and M. H. R. Stanley. We also thank M. Barthélemy, J.-P. Bouchaud, D. Sornette, D. Stauffer, S. Solomon, and J. Voit for helpful dicussions and comments.

\section{REFERENCES}

1. L. Bachelier, Ann. Sci. École Norm. Sup. 3, 21 (1900).

2. V. Pareto, Cours d'Économie Politique, Lausanne and Paris, 1897.

3. P. Lévy, Théorie de l'Addition des Variables Aléatoires, Gauthier-Villars, Paris, 1937.

4. B. B. Mandelbrot, J. Business 36, 294 (1963).

5. J. P. Bouchaud and M. Potters, Theorie des Risques Financiéres, Alea-Saclay, Eyrolles, 1998.

6. R. N. Mantegna and H. E. Stanley, An Introduction to Econophysics: Correlations and Complexity in Finance, Cambridge University Press, Cambridge 1999.

7. I. Kondor and J. Kértesz (eds) Econophysics: An Emerging Science, Kluwer, Dordrecht, 1999.

8. R. N. Mantegna (ed), Proceedings of the International Workshop on Econophysics and Statistical Finance, Physica $A$ [special issue] 269, 1 (1999). 
9. K. B. Lauritsen (ed), Application of Physics in Financial Analysis, Int. J. Theor. Appl. Finance [special issue] $\mathbf{x x}(1999)$.

10. T. Lux and M. Marchesi, Nature 297, 498 (1999); T. Lux, J. Econ. Behav. Organizat. 33, 143 (1998) ; J. Econ. Dyn. Control 22, 1 (1997); Appl. Econ. Lett. 3, 701 (1996).

11. M. Levy, H. Levy and S. Solomon, Economics Letters 45, 103 (1994); M. Levy and S. Solomon, Int. J. Mod. Phys C 7, 65 (1996).

12. J.-P. Bouchaud and R. Cont, Eur. Phys. J. B 6, 543 (1998); R. Cont and J.-P. Bouchaud, cond-mat/9712318.

13. M. Potters, R. Cont, and J.-P. Bouchaud, Europhys. Lett. 41, 239 (1998).

14. J.-P. Bouchaud and D. Sornette, J. Phys. I (France) 4, 863 (1994); D. Sornette, A. Johansen, and J.-P. Bouchaud, J. Phys. I (France) 6, 167 (1996); D. Sornette and A. Johansen, Physica A 261, 581 (1998); D. Sornette, Physica A 256, 251 (1998); A. Johansen and D. Sornette, Risk 1, 91 (1999), cond-mat/9901035, cond-mat/9811292.

15. D. Stauffer, Ann. Phys.-Berlin 7, 529 (1998); D. Stauffer and T. J. P. Penna, Physica A 256, 284 (1998); D. Chowdhury and D. Stauffer, Eur. Phys. J. B 8, 477 (1999); I. Chang and D. Stauffer, Physica A 264 (1999) 1; D. Stauffer and T. J. P. Penna, Physica A 256, 284 (1998); D. Stauffer, P. M. C. de Oliveria and A. T. Bernardes, Int. J. Theor. Appl. Finance 2, 83 (1999).

16. M. Marsili and Y.-C. Zhang, Phys. Rev. Lett. 80, 2741 (1998); G. Caldarelli, M. Marsili, and Y.-C. Zhang, Europhys. Lett. 40, 479 (1997); S. Galluccio, G. Calderelli, M. Marsili, and Y.-C. Zhang, Physica A 245, 423 (1997); M. Marsili, S. Maslov, and Y.-C. Zhang, Physica A 253, 403 (1998); S. Maslov and Y.-C. Zhang, Physica A 262, 232 (1999); D. Challet and Y. C. Zhang, Physica A 256, 514 (1998).

17. P. Bak, M. Paczuski and M. Shubik, Physica A 246, 430 (1997).

18. R. N. Mantegna and H. E. Stanley, Nature 376, 46 (1995).

19. S. Ghashghaie, W. Breymann, J. Peinke, P. Talkner, and Y. Dodge, Nature 381, 767 (1996); see also R. N. Mantegna and H. E. Stanley, Nature 383, 587 (1996); Physica A 239, 255 (1997).

20. A. Arneodo, J.-F. Muzy and D. Sornette, Eur. Phys. J. B 2, 277 (1998).

21. M. Pasquini and M. Serva, cond-mat/9810232; cond-mat/9903334.

22. M. M. Dacorogna, U. A. Muller, R. J. Nagler, R. B. Olsen and O. V. Pictet, $J$. International Money and Finance 12, 413 (1993); see also the Olsen website at http://www.olsen.ch for related papers.

23. R. Cont, Statistical Finance: Empirical study and theoretical modeling of price variations in financial markets, $\mathrm{PhD}$ thesis, Universite de Paris XI, 1998; (condmat/9705075); R. Cont, M. Potters, and J.-P. Bouchaud, in Scale Invariance and Beyond, edited by B. Dubrulle, F. Graner, and D. Sornette, Springer, Berlin, 1997.

24. N. Vandewalle and M. Ausloos, Int. J. Mod. Phys. C 9, 711 (1998); Eur. Phys. J. B 4, 257 (1998); N. Vandewalle, P. Boveroux, A. Minguet, and M. Ausloos, Physica A 255, 201 (1998).

25. H. Takayasu, A. H. Sato, and M. Takayasu, Phys. Rev. Lett. 79, 966 (1997); H. Takayasu, H. Miura, T. Hirabayashi, and K. Hamada, Physica A 184, 127 (1992).

26. K. N. Ilinski and A. S. Stepanenko, cond-mat/9806138; cond-mat/9902046; K. N. Ilinski, cond-mat/9903142.

27. A. Pagan, J. Empirical Finance 3, 15 (1996). 
28. G. W. Kim and H. M. Markowitz, J. Portfolio Management 16, 45 (1989); E. J. Elton and M. J. Gruber, Modern Portfolio Theory and Investment Analysis, J. Wiley, New York, 1995.

29. J.-P. Bouchaud, cond-mat/9806101; D. F. Wang, cond-mat/9807066, condmat/9809045; J.-P. Bouchaud, G. Iori, D. Sornette, Risk 93, 61 (March 1996); M. Rosa-Clot, S. Taddei, cond-mat/9901277, cond-mat/9901279; B. E. Baaquie, cond-mat/9708178; R. Cont, cond-mat/9902018.

30. F. Black and M. Scholes, J. Political Economy 81, 637 (1973).

31. R. N. Mantegna, Physica A 179, 232 (1991); J. Skjeltrop, University of Oslo Thesis, Oslo, Norway, 1998.

32. R. N. Mantegna and H. E. Stanley, Phys. Rev. Lett. 73, 2946 (1994).

33. I. Koponen, Phys. Rev. E 52, 1197 (1995).

34. P. Gopikrishnan, M. Meyer, L. A. N. Amaral and H. E. Stanley, Eur. Phys. J. B, 3139 (1998), cond-mat/9803374; P. Gopikrishnan, V. Plerou, L. A. N. Amaral, M. Meyer and H. E. Stanley, cond-mat/9905305, Phys. Rev. E., submitted.

35. M. M. Dacorogna, U. A. Muller, O. V. Pictet, and C.G. de Vries (Olsen group, www.olsen.ch, 199x).

36. Z. Ding, C. W. J. Granger and R. F. Engle, J. Empirical Finance 1, 83 (1983); For foreign exchange markets the decay time is even smaller [22].

37. E.-F. Fama, J. Finance 25, 383 (1970).

38. T. Bollerslev, R. Y. Chou, and K. F. Kroner, J. Econometrics 52, 5 (1992); G. W. Schwert, J. Finance 44, 1115 (1989); A. R. Gallant, P. E. Rossi and G. Tauchen, Review of Financial Studies 5, 199 (1992); B. Le Baron, J. Business 65, 199 (1992); K. Chan, K. C. Chan and G. A. Karolyi, Review of Financial Studies 4, 657 (1991).

39. C. W. J. Granger and Z. Ding, J. Econometrics 73, 61 (1996).

40. Y. Liu, P. Cizeau, M. Meyer, C.-K. Peng, and H. E. Stanley, Physica A 245, 437 (1997).

41. Y. Liu, P. Gopikrishnan, P. Cizeau, M. Meyer, C.-K. Peng and H. E. Stanley, Phys. Rev. E 60, xxx (1999), cond-mat/9903369.

42. P. Cizeau, Y. Liu, M. Meyer, C.-K. Peng, and H. E. Stanley, Physica A 245, 441 (1997).

43. J. Beran, Statistics for Long-Memory Processes (Chapman \& Hall, NY, 1994).

44. R. F. Engle, Econometrica 50, 987 (1982).

45. R. Ballie, J. Econometrics 17, 223 (1991) .

46. S. Galluccio, J.-P. Bouchaud, and M. Potters, Physica A 259, 449 (1998); L. Laloux, P. Cizeau, J.-P. Bouchaud and M. Potters, cond-mat/9810255; Risk 3, 69 (March, 1999).

47. V. Plerou, P. Gopikrishnan, B. Rosenow, L. A. N. Amaral, and H. E. Stanley, condmat/9903369.

48. E. P. Wigner, Ann. Math. 53, 36 (1951); E. P. Wigner, in Conference on Neutron Physics by Time-of-flight (Gatlinburg, Tennessee, 1956).

49. F. J. Dyson, J. Math. Phys. 3, 140 (1962); F. J. Dyson and M. L. Mehta, J. Math. Phys. 4, 701 (1963); M. L. Mehta and F. J. Dyson, J. Math. Phys. 4, 713 (1963).

50. T. Guhr, A. Müller-Groeling, and H. A. Weidenmüller, Phys. Rep. 299, 190 (1998); M. L. Mehta, Random Matrices, Academic Press, Boston, 1991. 
51. A. M. Sengupta and P. P. Mitra, cond-mat/9709283.

52. M. H. R. Stanley, L. A. N. Amaral, S. V. Buldyrev, S. Havlin, H. Leschhorn, P. Maass, M. A. Salinger, and H. E. Stanley, Nature 379, 804 (1996); L. A. N. Amaral, S. V. Buldyrev, S. Havlin, H. Leschhorn, P. Maass, M. A. Salinger, H. E. Stanley, and M. H. R. Stanley, J. Phys. I (France) 7, 621 (1997).

53. Y. Lee, L. A. N. Amaral, D. Canning, M. Meyer, and H. E. Stanley, Phys. Rev. Lett. 81, 3275 (1998).

54. V. Plerou, L. A. N. Amaral, P. Gopikrishnan, M. Meyer, and H. E. Stanley, Nature, in press, cond-mat/9906229.

55. S. V. Buldyrev, L. A. N. Amaral, S. Havlin, H. Leschhorn, P. Maass, M. A. Salinger, H. E. Stanley and M. H. R. Stanley, J. Phys. I (France) 7, 635 (1997).

56. L. A. N. Amaral, S. V. Buldyrev, S. Havlin, M. A. Salinger and H. E. Stanley, Phys. Rev. Lett. 80, 1385 (1998).

57. H. Takayasu and K. Okuyama, Fractals 6, 67 (1998). 